\title{
Electron Capture Ionization of Explosives with a Microflow Rate Particle Beam Interface
}

\author{
Achille Cappiello and Giorgio Famiglini \\ Istituto di Scienze Chimiche, Università di Urbino, Piazza Rinascimento 6, 61029 Urbino, Italy \\ Antonietta Lombardozzi, Alessandro Massari, and Gianni Giulio Vadalà \\ Direzione Centrale Polizia Criminale, Servizio Polizia Scientifica, Viale dell'Arte 38, 00144 Rome, Italy
}

\begin{abstract}
A method for the analysis of four widely used explosives based on reversed-phase liquid chromatography coupled to a quadrupole mass spectrometer is presented. A microflow rate particle beam interface was employed that offers simplified operation procedures and improved interfacing performance. A positive role played by the reduced size of the aerosol droplets generated by the microflow rate interface is outlined in this work. Greater vaporization efficiency and negligible thermal decomposition were observed for the selected compounds in the ion source of the mass spectrometer. Electron capture ionization allowed specific and sensitive determination of the analytes. Detection limits that ranged between 60 and $200 \mathrm{pg}$ and had a signal-to-noise ratio of 5:1 were obtained in selected ion monitoring mode after column elution. (J Am Soc Mass Spectrom 1996, 7, 753-758)
\end{abstract}

$\mathrm{T}$ The determination of specific explosives in various matrices at trace-level concentration continues to be of great importance in both forensic and environmental applications. The unambiguous identification of a certain compound in postexplosion residues may help to focus an investigation in a particular direction. Furthermore, the analytical results could be used as evidence in a judicial proceedings. Also, the recent massive reduction of ammunition storage and production has increased the need to monitor the soil plus surface and underground water in the vicinity of dismantled plants and warehouses. The large spectrum of samples in which explosives need to be detected requires very sensitive and selective analytical techniques.

Due to the chemical instability of explosives, liquid chromatography is often the technique of choice to separate explosives from other interferences. The combination of liquid chromatography and mass spectrometry provides an unmatched capability for the analysis of nonvolatile and thermally unstable compounds. Yinon and co-workers [1-3] have contributed greatly to the development of several methods for the analysis of explosive compounds via various liquid chromatography-mass spectrometry (LC-MS) coupling techniques. Direct liquid introduction (DLI) was the first interface to be used for this application [1]. The necessity of mobile phase splitting considerably impaired

Address reprint requests Achille Cappiello, Istituto di Scienze Chimiche, Università di Urbino, Piazza Rinascimento 6, 61029 Urbino, Italy. the sensitivity, but, on the other hand, it offered a valuable first approach. The thermospray interface also was employed for the analysis of explosives in both positive and negative ion mode [3]. The availability of a gentle ionization technique allowed molecular weight information to be obtained. Thermospray also showed some limitations with respect to the mobile phase composition. The presence of several adduct ions in the mass spectrum limited detection sensitivity. Among the growing family of LC-MS interfaces, the particle beam interface represents the last refuge for electron impact ionization. For this reason it stands apart in this group. The possibility to produce reproducible and library searchable mass spectra makes it particularly suitable for the analysis of small molecules. Recently, several improvements have contributed to access of the full potential of this technique [4-8]: increased sensitivity, superior compatibility with aqueous mobile phases, easier tuning procedures, and improved transport of the solute to the ion source.

In this work we evaluate the possibility to analyze a few selected explosives with a particle beam interface. The instrumentation employed took advantage of all the above-mentioned improvements. Because of the chemical composition of the explosive considered, electron capture ionization was implemented [9]. Typical fragmentation patterns are discussed. Instrument conditions were optimized for the best sensitivity, specificity, and reproducibility. Vaporization and ionization conditions were also optimized to minimize thermal decomposition phenomena and the possibility of ion-molecule reactions. A reversed-phase $250-\mu \mathrm{m}-$ i.d. C18 packed capillary column was used for the 
separation of explosives. The column was coupled to a modified nebulizer that was developed in our laboratory and is capable of handling complete nebulization from a mobile phase flow rate as low as $1 \mu \mathrm{L} / \mathrm{min}$ $[4,5]$. This characteristic allows operation at near ambient temperature in the desolvation chamber and reduces the amount of solvent vapors transferred to the ion source. This situation is particularly advantageous during electron capture ionization where contaminants may deplete available electrons and thus reduce sample ionization rate and increase chances of adduct ion formation. Negative ion ionization, carried out with the modified interface, allowed specific and sensitive identification of the selected explosives at picogram level with only negligible thermal decomposition. A comparison of the mass spectral results obtained with a conventional interface also is reported.

\section{Experimental}

\section{Mass Spectrometer and Particle Beam Interface}

All the experiments were carried out with a HewlettPackard (Palo Alto, CA) 59980B particle beam unit coupled with a Hewlett-Packard 5989A mass spectrometer. The original nebulizer was replaced by a laboratory-made microflow nebulizer $[4,5]$ that generates a mobile phase aerosol with flow rates as low as 1 $\mu \mathrm{L} / \mathrm{min}$. A $50-\mu \mathrm{m}$-i.d., 180- $\mu \mathrm{m}$-o.d. fused silica capillary tubing (Polymicro Technologies, Phoenix, AZ) was used as the nebulizer tip and to connect the liquid chromatograph. The nebulizing gas was 5.6 purity grade ( $>99.9996 \%$ ) helium and was purchased from SOL (Milano, Italy). The helium pressure was 70-90 psi to supply $0.1 \mathrm{~L} / \mathrm{min}$ of gas. The desolvation chamber temperature was kept at $40{ }^{\circ} \mathrm{C}$. The operating pressure was 0.5 torr in the desolvation chamber, 0.3 torr in the second stage of the momentum separator, and 8-10 $\times 10^{-5}$ torr in the manifold of the ion source. Pressure data were collected during standard electron impact (EI) ionization conditions and with the microflow interface in place. Electron capture ionization was carried out with methane used as the reagent gas. Methane, 4.5 purity grade ( $>99.995 \%$ ), was purchased from Union Carbide (Westerlo, Belgium). The gas was admitted into the ion source via the interface isolation valve. The solute particles and the reagent gas reached the ionization chamber through an entrance hole in the ion source plunger. The original hole was enlarged to 3 $\mathrm{mm}$ to admit a larger portion of the beam into the ion source. Higher local pressure conditions, required by chemical ionization, were obtained by switching to a dedicated ionization chamber. Preliminary mass spectrometer tuning and calibration were performed automatically in EI conditions by using perfluorotributylamine (PFTBA) as a reference compound and monitoring $m / z 69,219$, and 502 . The repeller potential was adjusted manually. Reagent gas pressure was optimized to maximize $m / z 41$ in the positive ion mode.
The higher response was obtained with a 1.8-torr methane pressure measured close to the ion source. Ramps of the main electric parameters were performed to optimize negative ionization conditions. PFTBA was introduced constantly during this procedure and negative ions at $m / z 302,452$, and 633 were monitored. The mobile phase was allowed into the ion source during calibration. The dwell times during selected ion monitoring (SIM) analyses were adjusted to obtain 0.5 cycles per second and a mean of 10 acquisition samples for each high-performance liquid chromatography (HPLC) peak. The ions used in SIM mode were collected in a single ion program. The final transfer tube was extended fully toward the source block. The ion source temperature was set at $150{ }^{\circ} \mathrm{C}$; this value was the best compromise between vaporization requirements and electron capture conditions.

\section{Liquid Chromatography}

Liquid chromatography was carried out with a Kontron Instrument 420 dual-pump, binary-gradient, conventional HPLC system (Kontron Instrument, Milano, Italy). Microliter flow rates were obtained with a laboratory-made splitter that was placed between the pumping system and the injector [10]. For sample injections, a Valco injector (Valco, Houston, TX) equipped with internal loops $(0.06$ and $0.5 \mu \mathrm{L})$ was employed. A laboratory-made packed capillary column was used for the chromatographic separation [11]. These columns are routinely made in our laboratory from 1/16-in.-o.d., 250- $\mu$ m-i.d. PEEK tubing (Alltech Associates Inc., Deerfield, IL) and are packed with reversed-phase 5- $\mu \mathrm{m}$ particle size $\mathrm{C} 18$ purchased from Phase Sep (Queensferry, UK). A 25-cm-long column has a mean of 20.000 theoretical plates at 1$\mu \mathrm{L} / \mathrm{min}$ flow rate. Acetonitrile was used as an organic solvent in the mobile phase. Acetonitrile was preferred to methanol because of its lower viscosity. Relative solvent concentration in the mobile phase, its flow rate, and gradient programs are described in detail in the Results and Discussion section.

\section{Reagents}

All solvents were HPLC grade from Farmitalia Carlo Erba (Milano, Italy) and were filtered and degassed before use. Reagent water was obtained from a Milli-Q water purification system (Millipore Corp., Bedford, MA).

\section{Results and Discussion}

The explosives used for this study, 2,4,6-trinitrotoluene (TNT), pentaerythritol tetranitrate (PETN), 1,2,3-propanetriol trinitrate or nitroglycerin (NG), and 1,3,5trinitro-1,3,5-triazacyclohexane (RDX), contain a large number of nitro groups in their structure (Figure 1). This characteristic make them particularly suitable to negative ionization. 


$$
\begin{aligned}
& \mathrm{CH}_{2}-\mathrm{ONO}_{2} \\
& \mathrm{C} \mathrm{H}-\mathrm{ONO}_{2} \\
& 1 \\
& \mathrm{CH}_{2}-\mathrm{ONO}_{2}
\end{aligned}
$$

1, 2,3-Propanetriol trinitrate (nitrogiycerín)<smiles>O=[N+]([O-])CC(CO[N+](=O)[O-])(CO[N+](=O)[O-])CO[N+](=O)[O-]</smiles>
Pentaerythritol tetranitrate (PETY)<smiles>Cc1c([N+](=O)[O-])cc([N+](=O)[O-])cc1[N+](=O)[O-]</smiles>

2,4,6-Mrinitrotoluene (TNT)

1,3,5-Trinitro-1, 3,5-Triazacyclohexane<smiles>O=[N+]([O-])N1CN([N+](=O)[O-])CN([N+](=O)[O-])C1</smiles>

Figure 1. Structural formula of selected explosives.

A particle beam interface requires a high temperature vaporization of molecular aggregates prior to the ionization process. The molecular aggregates originate from the solute, dissolved in aerosol droplets. As pointed out by Jones et al. [12] and also discussed in recent papers $[6,13]$, the way heat is transferred to the solute particles and how the particles interact with the hot source surface is still a question. The vaporization process is tolerated poorly by many heat-sensitive compounds, and explosives fall into this category. Considerable experience in the nebulization of HPLC eluates enabled our research group to develop a device specifically designed for microcolumn flow rates (1-5 $\mu \mathrm{L} / \mathrm{min}$ ). One of the unforeseen advantages of the scaled-down nebulizer is a consistent reduction of the aerosol droplet size to the submicrometer range. This feature facilitates the formation of smaller solute particles with much higher surface-to-mass ratio. Once the particles impact the source surface, the heat is distributed faster throughout the whole particle, which produces very efficient evaporation of molecules without decomposition. Additionally, with conventional mobile phase flow rates of about $500 \mu \mathrm{L} / \mathrm{min}$, larger amounts of solvent vapors are admitted into the ion source. Oxygen and other electronegative species that act like electron traps can be found in these contaminants: they lower analyte ionization rate and decrease sensitivity during electron capture ionization. For all these reasons a microflow rate interface was employed in this work.

Figure 2 shows the mass spectra obtained by electron capture ionization of $300 \mathrm{ng}$ of an explosives standard solution injected via the modified particle beam interface. The experiment was carried out by flow injection analyses (FIA) with a mobile phase composed of an equal concentration of water and acetonitrile. The mobile phase flow rate was $1 \mu \mathrm{L} / \mathrm{min}$. TNT
(Figure 2a), the most chemically stable in this group, shows a strong molecular ion $\left[\mathrm{M}^{-}\right]$at $\mathrm{m} / z 227$ with some fragmentation. Interesting losses of $30[\mathrm{M}-$ $\mathrm{NO}^{-}$and $17[\mathrm{M}-\mathrm{OH}]^{-}$also can be observed. Solid probe sample introduction [15] gave similar results except for a more intense $[\mathrm{M}-\mathrm{H}]^{-}$signal. RDX (Figure $2 \mathrm{~b}$ ) shows a quite complex mass spectrum with a weak molecular ion $(<2 \%)\left[\mathrm{M}^{-}\right]$at $m / z \quad 222$ and $[\mathrm{M}-\mathrm{H}]^{-}$at $m / z 221$ as already reported in a previous article [14]. Two intense adduct ions at $\mathrm{m} / \mathrm{z} 268$ $\left[\mathrm{M}+\mathrm{NO}_{2}\right]^{-}$and $m / z \quad 324$ are visible clearly in the spectrum. The ion signal at $m / z 324$ may originate from a reaction with part of the cyclic structure. The loss of $\mathrm{NO}_{2},\left[\mathrm{M}-\mathrm{NO}_{2}\right]^{-}$, produces a strong signal at $m / z$ 176. Intense ion signals at $m / z 129$ and 102 , the base peak, originated from the decomposition of the cyclic structure. PETN and NG (Figure $2 \mathrm{c}$ and d) show similar spectra with an intense signal at $m / z 62\left(\mathrm{NO}_{3}^{-}\right)$, $m / z 289$ for NG, and $m / z 378$ for PETN $\left[\mathrm{M}+\mathrm{NO}_{3}\right]^{-}$. No molecular ion information were found in their spectra. The ratio between $\mathrm{NO}_{3}^{-}$and $\left[\mathrm{M}+\mathrm{NO}_{3}\right]^{-}$ intensity in PETN and NG mass spectra varies in accordance with the amount of sample injected into the system. $\left[\mathrm{M}+\mathrm{NO}_{3}\right]^{-}$is produced from an ionmolecule reaction in the gas phase promoted by the higher concentration of reagents in chemical ionization $\mathrm{CI}$ conditions. The abundance of the $\left[\mathrm{M}+\mathrm{NO}_{3}\right]^{-}$ion exceeds the $\mathrm{m} / \mathrm{z} 62$ intensity at high concentrations.

Linear regression plots for concentration calibration were obtained for all explosives in a concentration range from 20 to $100 \mathrm{ppm}$. The $\mathrm{m} / z 227$ was used for TNT, $m / z 129$ for RDX, and $m / z 62$ for NG and PETN. Plots were obtained from the ion profiles that average peak area counts of five injections for each concentration (0.06- $\mu \mathrm{L}$ loop). Mean standard deviations for the quantitation method were obtained from the same data and were $7.0 \%$ for TNT, $11.6 \%$ for RDX, $16.3 \%$ for NG, and $4.9 \%$ for PETN. Correlation coefficients confirmed appreciable response linearity for these compounds; these coefficients are reported in parentheses after the linear regression equations: $y=$ $5.1 x-8.9(0.998)$ for TNT, $y=3.6 x-65.1(0.999)$ for RDX, $y=1.35 x-19.5(0.999)$ for NG, and $y=0.9 x$ - 14.1 (0.991) for PETN.

Interesting insights with regard to solute vaporization in the particle beam interface can be obtained from a comparison between a conventional and a microflow rate nebulizer when nitroglycerin is analyzed. Figure 3 shows the ion profiles at $\mathrm{m} / z \quad 62$ and 289 obtained by two injections of $30 \mathrm{ng}$ of NG with a conventional nebulizer (Figure $3 \mathrm{a}$ ) and $3.6 \mathrm{ng}$ with a microflow rate nebulizer (Figure $3 b$ ). The concentration of the sample injected in the conventional interface was increased because of a consistent difference in the response sensitivity between the two interfaces. During the experiments the mobile phase was composed of equal amounts of water and acetonitrile. Flow rate was kept at $1 \mu \mathrm{L} / \mathrm{min}$ with the microflow rate interface and was increased up to $400 \mu \mathrm{L} / \mathrm{min}$ with the 


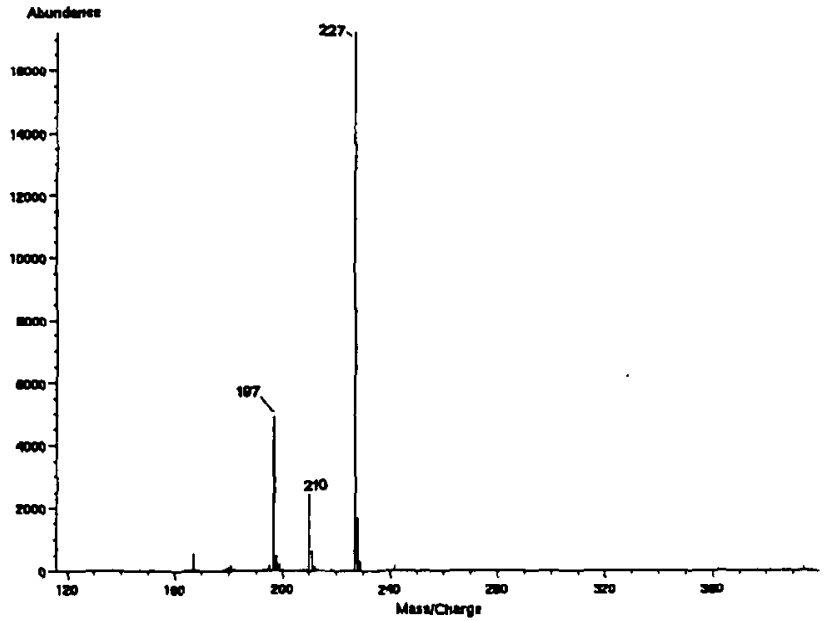

(a)

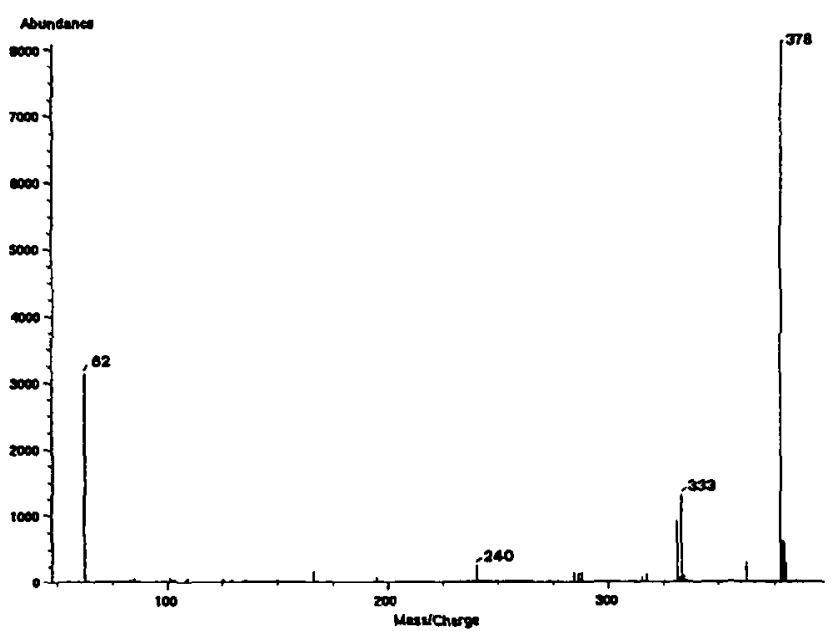

(c)

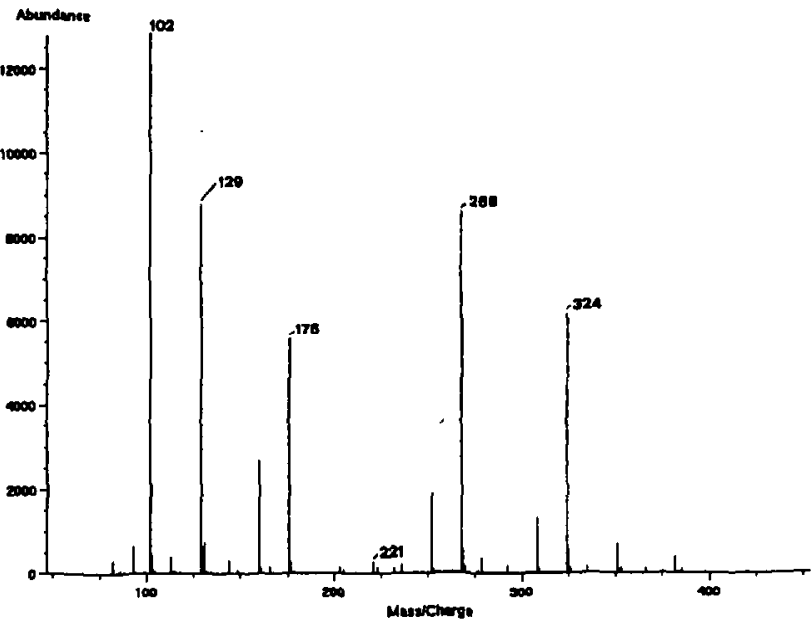

(b)

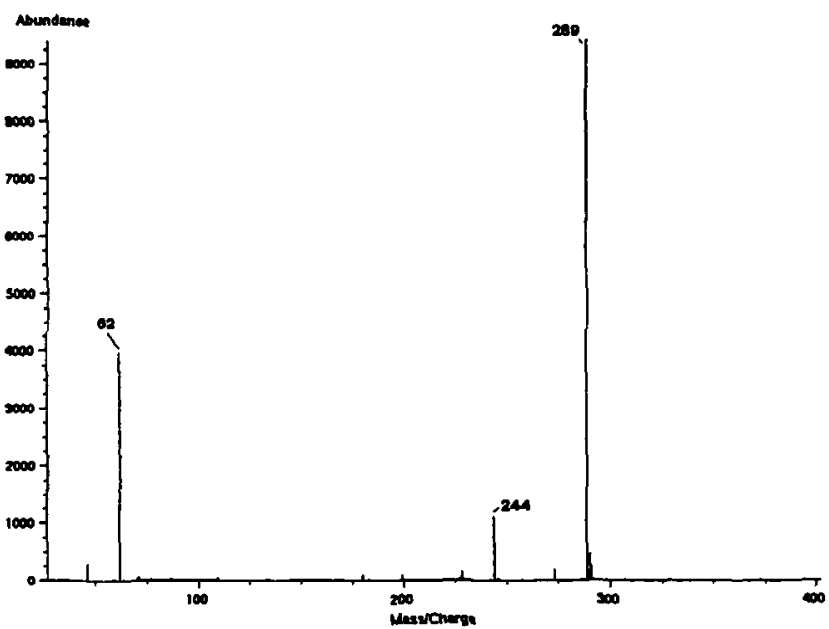

(d)

Figure 2. Electron capture mass spectra: (a) INT; (b) RDX; (c) PETN; (d) NG.

conventional interface. 0.06- and $0.5-\mu \mathrm{L}$ internal loops were employed with the micro and conventional interfaces, respectively. It is clearly apparent from the signal profiles that different vaporization mechanisms occur in the ion source when different nebulizations are used. The heavier solute particles that were produced by the conventional interface showed an initial boost of vaporization immediately after the impact with the hot target surface inside the ion source. This phenomenon was confirmed by the corresponding increase of $\mathrm{m} / \mathrm{z} 289$ and 62 signals that prove the concurrent presence of $\mathrm{NO}_{3}^{-}$and the intact molecules in the gas phase. Subsequent decomposition occurred as demonstrated by the second broad peak at $\mathrm{m} / \mathrm{z} 62$ without any corresponding response at $m / z$ 289. In this case, $m / z 62$ indicates thermal decomposition of the molecular structure to generate $\mathrm{NO}_{3}$. Lack of intact molecules in the gas phase precluded a $\mathrm{m} / \mathrm{z} 289$ ion signal. The smaller and lighter solute particles with a higher surface-to-mass ratio that is produced by the micronebulizer allow complete vaporization of intact molecules of nitroglycerin as indicated by the simultaneous rise of $m / z 62$ and 289 ion signals. Flash vapor- ization converts all the substances to the gas phase with an increased ionization efficiency and higher sensitivity.

Detection limits were evaluated in SIM mode by using a packed capillary column for peak retardation. Limits were based on a signal-to-noise ratio of 5:1 and were $60 \mathrm{pg}$ for NG, $120 \mathrm{pg}$ for TNT, and $200 \mathrm{pg}$ for RDX and PETN.

Figure 4 shows the chromatographic separation of the four explosives obtained with a packed capillary column in gradient conditions. Mobile phase composition was changed from 60:40 water:acetonitrile to 20:80 in $20 \mathrm{~min}$. The mobile phase flow rate was kept at 2 $\mu \mathrm{L} / \mathrm{min}$. The ion selection was based on specificity and abundance criteria. Although less specific than other high-mass adduct ions present in the mass spectra, the negative fragment ion at $m / z 62$, which was selected for the NG and PETN detection, showed a linear calibration response and its relative abundance was not influenced by the sample concentration in the ion source.

Another aspect involved in the analysis of explosives with a particle beam interface is the mobile phase 


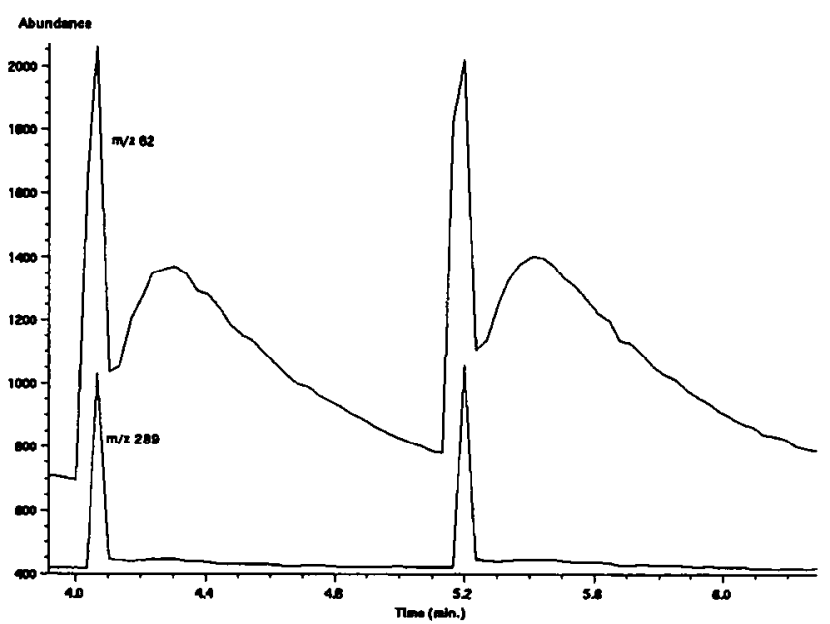

(a)

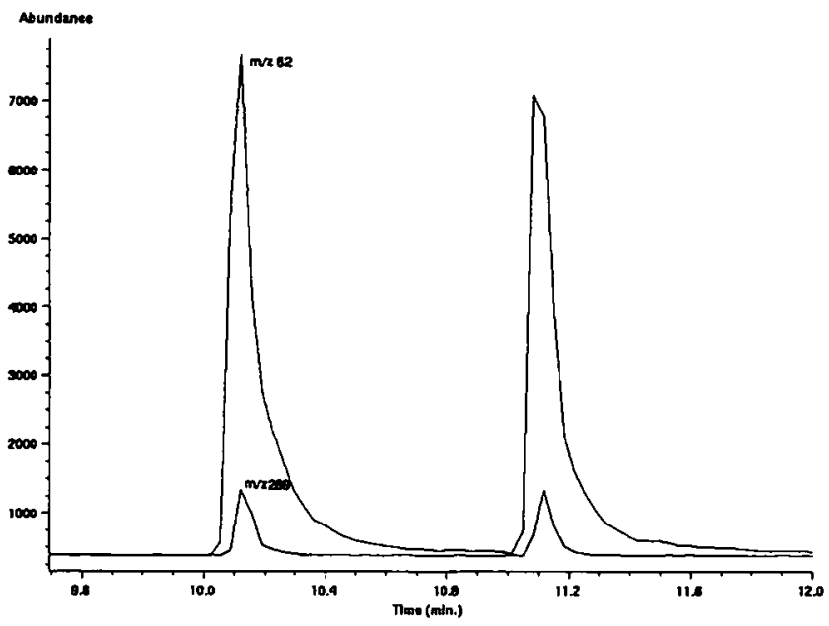

(b)

Figure 3. $m / z \quad 62$ and 289 ion profiles of two consecutive injections of NG: (a) conventional interface; (b) microflow rate interface.

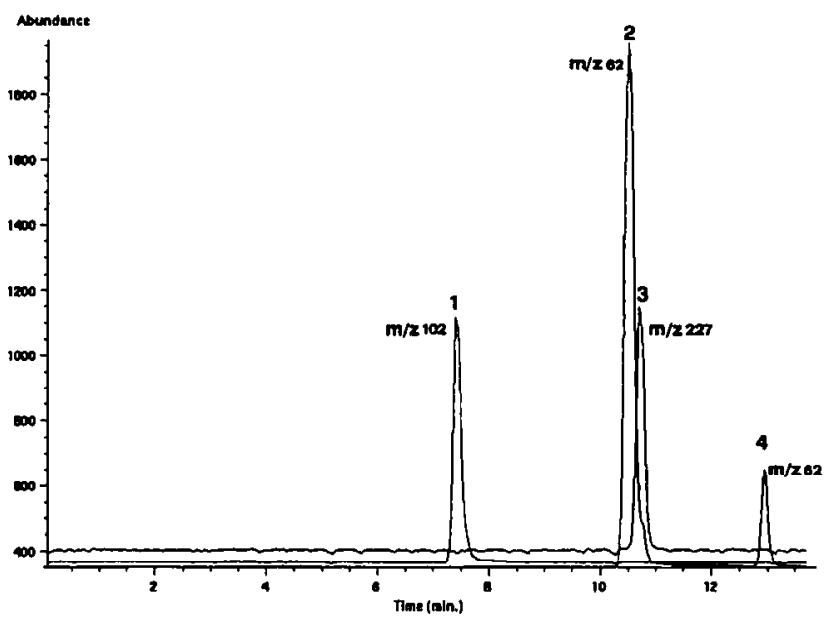

Figure 4. Chromatographic separation of selected explosives: (1) RDX; (2) NG; (3) TNT; (4) PETN.

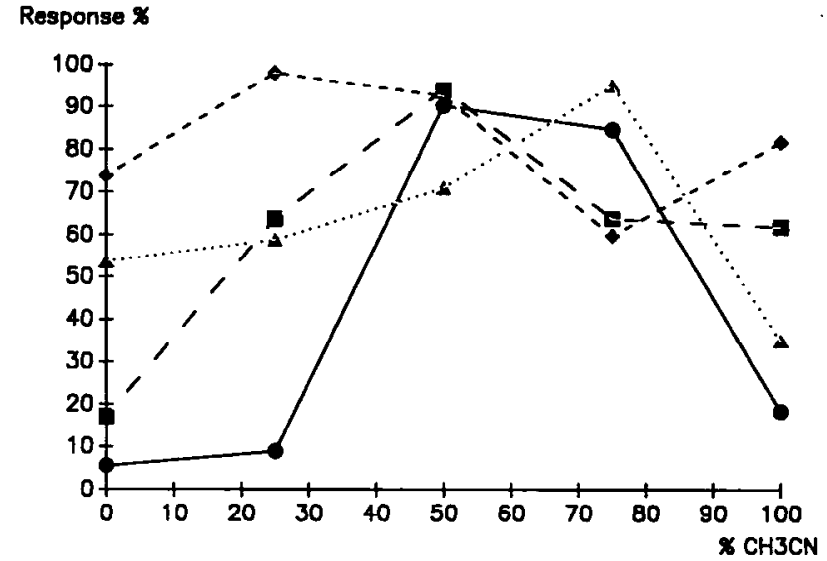

Figure 5. Normalized response obtained from the analysis of explosives with different relative concentrations of water and acetonitrile: , NG; $\boldsymbol{\square}, \mathrm{RDX} ; \Delta, \mathrm{PETN} ; \bullet, \mathrm{TNT}$.

composition. The effects of different solvents all along the sequence of different processes from nebulization to vaporization may impair the quality of analytical results during gradient analysis. As discussed earlier in this section, solvent vapors that escape from the double pumping stage of the momentum separator may contaminate the ion source environment, which worsens chemical ionization results. In reversed-phase liquid chromatography a combination of water and organic solvents is used. Relative concentrations may vary consistently with noticeable effects on the ionization behavior. Solvent intake with a microflow rate nebulizer is minimal. Because of the fact that even a trace of an electronegative species may deplete available electrons in negative ionization, different mobile phase composition were evaluated for the analysis of explosives. Relative concentrations of water and acetonitrile were changed from 0 to $100 \%$ by $25 \%$ increments. For each composition the selected explosives were injected and relative peak areas were recorded. The results were normalized and are reported in Figure 5. It is evident that each compound reacts differently to a specific mobile phase composition, but all of the compounds show the highest response at the middle of the range. NG showed reduced sensitivity at both extremes of the range. Sensitivity for RDX decreased at high concentrations of water, whereas PETN showed decreased response at $100 \%$ acetonitrile. TNT showed an almost flat response over the complete range. Perhaps a combination of different phenomena such as nebulization, transport, and ionization contribute to the observed behavior. Current mobile phase gradients for the separation of explosives would not affect performance significantly.

These results indicate that poor nebulization of HPLC eluate is responsible to a great extent for poor mass spectral results when chemically delicate compounds are considered. Further studies on alternative surface materials that are compatible with electron capture ionization have to be completed. Such studies 
hopefully will supply other information on the applicability of the particle beam to other compound classes.

In conclusion, explosives are a good example of compounds that can benefit immensely from the microflow rate nebulization. Particle beam interface in this particular configuration showed several advantages compared to a conventional interface when alternative ionization techniques are involved. Electron capture ionization allowed specific and sensitive determination of explosives and certainly may contribute to their identification at trace-level concentrations.

\section{References}

1. Yinon, J.; Hwang, D. G. J. Chromatogr. 1983, 268, 45-53.

2. Yinon, J.; Hwang, D. G. J. Chromatogr. 1985, 339, 127-137.

3. Voyksner, R. D.; Yinon, J. J. Chromatogr. 1986, 354, 393-405.

4. Cappiello, A.; Bruner, F. Anal. Chem. 1993, 65, 1281.
5. Cappiello, A.; Famiglini, G. Anal. Chem. 1994, 66, 3970.

6. Cappiello, A.; Famiglini, G. Anal. Chem. 1995, 67, 412.

7. Bellar, T. A.; Behymer, T. D.; Budde, W. L. J. Am. Soc. Mass Spectrom. 1990, 1, 92-98.

8. Ho, J. S.; Behymer, T. D.; Budde, W. L.; Bellar, T. A. J. Am. Soc. Mass Spectrom. 1992, 3, 662-671.

9. Hunt, D. F.; Crow, F. W. Anal. Chem. 1978, 50, 1781-1784.

10. Berloni, A.; Cappiello, A.; Famiglini, G.; Palma, P. Chromatographia 1994, 39, 279.

11. Cappiello, A.; Palma, P.; Mangani, F. Chromatographia 1991, $32,389$.

12. Jones, G. G.; Pauls, R. E.; Willoughby, R. C. Anal. Chem. 1991, $63,460-463$,

13. Betowski, L. D.; Pace, C. M.; Roby, M. R. I. Am. Soc. Mass Spectrom. 1992, 3, 823-830.

14. McLuckey, S. A.; Glish, G. L.; Carter, J. A. I. Forensic Sci. 1985, 30, 773.

15. McLuckey, S. A.; Glish, G. L.; Kelley, P. E. Anal. Chem. 1987, $59,1670$. 